\title{
Reconstrução de defeitos ósseos no complexo craniofacial por meio de próteses individualizadas - estudo piloto
}

\section{Reconstruction of craniofacial bone defects with customized implants - a pilot study}

\author{
Ferdinando De Conto \\ Bruna Laíz Dagort* \\ Luiz Airton Consalter** \\ Liciane Sabadin Bertol ${ }^{* * * *}$ \\ Camila De Conto ${ }^{* * * * *}$ \\ Jurriaan Kloek $k^{* * * * *+}$
}

\section{Resumo}

A Prototipagem Rápida tem demonstrado ser uma ferramenta muito útil no auxílio ao desenvolvimento de novos produtos na área biomédica. Essa tecnologia permite a obtenção de protótipos físicos a partir da conversão de imagens obtidas de tomografia computadorizada. Objetivo: O objetivo deste estudo piloto foi testar a precisão do processo de prototipagem por método de impressão tridimensional. Materiais e método: Foram confeccionados biomodelos de próteses individualizadas produzidas por método de espelhamento gráfico para reconstrução de defeitos ósseos no complexo craniofacial criados em crânio seco de humano. Resultados: Comparando ambas as medidas notou-se que não houve diferença significativa, muito menos um desvio considerável, quando comparadas graficamente as peças originais com os seus respectivos protótipos. Conclusão: Este estudo revela que o método de espeIhamento gráfico para obtenção de um biomodelo e a sua confecção por prototipagem rápida pode ser uma alternativa terapêutica viável por apresentar fiéis reconstruções, uma vez que este método utiliza o próprio individuo como modelo.

Palavras-chave: Tomografia. Imagem tridimensional. Modelos anatômicos.

\section{Introdução}

Estudos vêm sendo realizados para elaborar novas alternativas para reconstrução de grandes defeitos no complexo craniofacial. Uma dessas alternativas é a confecção de próteses individualizadas por Prototipagem Rápida (PR), técnica que surgiu no final dos anos 80, que encurta o procedimento cirúrgico, com menores danos ao paciente; proporciona melhor prognóstico pós-operatório e o retorno da função e estética ${ }^{1-3}$.

O protótipo é um produto fabricado unitariamente, segundo as especificações de um projeto, com a finalidade de servir de teste, antes da sua fabricação em escala industrial. Em outras palavras, pode-se dizer que o protótipo é um experimento virtual que tenta imitar um sistema real' ${ }^{1}$ Os biomodelos de PR são protótipos biomédicos obtidos a partir de imagens de tomografia computadorizada (TC) ou ressonância nuclear magnética $(\mathrm{RNM})$ passíveis de serem utilizados com objetivos didáticos na fabricação de implantes protéticos, no diagnóstico precoce e no tratamento de deformidades faciais, além

Doutor - Faculdade de Odontologia - Universidade de Passo Fundo, Passo Fundo - RS, Brasil.

Cirurgiã Dentista - Faculdade de Odontologia - Universidade de Passo Fundo, Passo Fundo - RS, Brasil.

Doutor - Faculdade de Engenharia Mecânica - Universidade de Passo Fundo, Passo Fundo - RS, Brasil.

Doutor - Faculdade de Engenharia Mecânica - Universidade Federal do Rio Grande do Sul, Porto Alegre - RS, Brasil.

Engenheira de Materiais - Eccole Européenne d'ingénieurs en Génie des Matériaux - Université de Lorraine, Nancy, Paris.

Mestre - Department of Fusion Physics and Engineering - Université de Lorraine, Nancy, Paris. 
de facilitar, também, a comunicação entre a equipe profissional e o paciente ${ }^{2,4}$.

Os biomodelos permitem a mensuração de estruturas, a simulação de osteotomias e técnicas de ressecção, além de um completo planejamento dos mais diversos tipos de cirurgias na região bucomaxilofacial, o que tende a reduzir o tempo do procedimento cirúrgico e, consequentemente, o período de anestesia, bem como o risco de infecção, havendo, ainda, melhora no resultado e na diminuição no custo global do tratamento. Na produção dos biomodelos, é necessário o processamento de um grande volume de dados e uso intensivo da informática. Pela natureza demorada desse processo, associada ao custo do biomodelo, sua aplicação é dificultada em procedimentos cirúrgicos de rotina, limitação essa que tende a ser minimizada pelo avanço tecnológico e pela utilização interdisciplinar da tecnologia de prototipagem ${ }^{3,5-7}$.

Tal sistema gera a peça a partir da união gradativa do material, camada por camada. É uma tecnologia que permite produzir modelos e protótipos a partir de um modelo sólido de reconstrução volumétrica tridimensional (3D) no sistema CAD (computer-aided design) e CAM (computer-aided manufacturing $)^{8}$. A integração dos sistemas CAD aos sistemas médicos facilita a manipulação e modelagem de objetos, permitindo que imagens virtuais de estruturas segmentadas possam ser manipuladas como se fossem peças de um quebra-cabeça ou de um protótipo biomecânico. Além disso, sistemas CAD são adequados para definir procedimentos de espelhamento, utilizando a simetria contralateral da face, permitindo o planejamento e a obtenção de próteses personalizadas, inclusive com a simulação da montagem da prótese virtual no modelo $3 \mathrm{D}^{7}$.

Todos os processos de prototipagem rápida existentes atualmente são constituídos por cinco etapas básicas: criação de um modelo $\mathrm{CAD}$ da peça que está sendo projetada; conversão do arquivo CAD em formato STL, próprio para estereolitografia; divisão do arquivo STL em finas camadas transversais; construção física do modelo, empilhando-se uma camada sobre a outra; limpeza e acabamento do protótipo? ${ }^{9}$.

Os principais sistemas de prototipagem rápida usados na fabricação de modelos estão descritos a seguir $^{9,10}$ :

\section{Estereolitografia (SLA, Stereolithography)}

Esse processo pioneiro, patenteado em 1986, deflagrou a revolução da prototipagem rápida. Ele constrói modelos tridimensionais a partir de polímeros líquidos sensíveis à luz, que se solidificam quando expostos à radiação ultravioleta. Uma vez que a estereolitografia foi a primeira técnica bem sucedida de prototipagem rápida ela se tornou um padrão de avaliação para as demais, que surgiram posteriormente ${ }^{7}$.

\section{Sinterização Seletiva a Laser (SLS, Selective Laser Sintering)}

Essa técnica, patenteada em 1989, usa um raio de laser para fundir, de forma seletiva, materiais pulverulentos, tais como náilon, elastômeros e metais, num objeto sólido. As peças são construídas sobre uma plataforma posicionada imediatamente abaixo da superfície de um recipiente preenchido com o pó fusível por calor. O raio laser traça a primeira camada, sinterizando o material. A plataforma é ligeiramente abaixada, reaplica-se o pó e o raio laser traça a segunda camada. $\mathrm{O}$ processo continua até que a peça esteja terminada. $\mathrm{O}$ pó em excesso ajuda a dar suporte ao componente durante sua construção $0^{10}$.

\section{Modelagem por Deposição de Material Fundido (FDM, Fused Deposition Modeling)}

Nesse processo filamentos de resina termoplástica aquecida são extraídos a partir de uma matriz em forma de ponta que se move num plano X-Y. A matriz de extrusão controlada deposita filetes de material muito fino sobre a plataforma de construção. A plataforma é mantida sob uma temperatura inferior à do material, de forma que a resina termoplástica endurece rapidamente. As resinas termoplásticas adequadas a esse processo incluem poliéster, polipropileno, acrilonitrila-butadieno- estireno (ABS), elastômeros e cera usada no processo de fundição por cera perdida ${ }^{11}$.

\section{Bioprototipagem, por meio da técnica da impressão tridimensional (3DPTM)}

Com esse método são construídas peças (camada por camada) de forma aditiva. A prototipagem rápida é muito utilizada nas indústrias automotiva e aeroespacial, de telecomunicações, de máquinas industriais e de eletrodomésticos. A impressão tridimensional pode usar diferentes tipos de materiais simultaneamente e apresenta menor custo de produção. Contudo, os modelos apresentam rugosidade elevada, relativa fragilidade e porosidade. A técnica de 3DPTM funciona como uma impressora de jato de tinta. Contudo, imprimindo em três dimensões ${ }^{11}$.

Este estudo piloto teve por objetivo testar a precisão do processo de prototipagem com uso de biomodelo de próteses individualizadas para reconstrução de defeitos ósseos no complexo craniofacial criado em crânio seco de humano, avaliando sua 
adaptação por medidas diretas e, por comparação gráfica, avaliando, também, a fidelidade da reprodução do protótipo.

\section{Materiais e método}

Este estudo foi realizado a partir de um crânio seco selecionado no Departamento de Morfologia da Universidade de Passo Fundo e Setor de Tomografia do Hospital da Cidade de Passo Fundo, mediante aprovação do Comitê de Ética em Pesquisa da Universidade de Passo Fundo sob o protocolo $\mathrm{n}^{\mathrm{o}}$ $316 / 2011$

\section{Marcação dos pontos e medidas craniométricas}

A mensuração foi feita com auxílio do paquímetro digital calibrado da marca Mitutoyo ${ }^{\circledR}$ (São Paulo, SP, Brasil) cada uma das medidas foi repetida 10 vezes pelo mesmo examinador. A ordem das mensurações foi aleatória e os resultados obtidos foram registrados em um quadro elaborado para tal fim.

Para as medidas foram utilizados os seguintes pontos e medidas craniométricas (Fig. 1):

- FZ: ponto situado na extremidade medial da sutura frontozigomática, bilateralmente;

- Zy: ponto situado na extremidade lateral do arco zigomático, bilateralmente;

- MAE: ponto localizado na extremidade lateral do meato acústico externo, bilateralmente;

- FSO: ponto situado no forame supra orbitário, bilateralmente;

- PMZ: ponto localizado no pilar maxilo zigomático, bilateralmente;

- Largura bizigomática (LBZ): distância entre os pontos Zy direito e esquerdo;

- Largura do zigomático ao meato acústico externo (ZMA): distância dos pontos Zy e MAE direito e esquerdo;

- Largura do pilar maxilo zigomático ao forame supra orbitário (PFS): distância dos ponto PMZ e FSO direito e esquerdo;

- Frontozigomático (FZ-FZ): distância entre os pontos FZ direito e esquerdo.
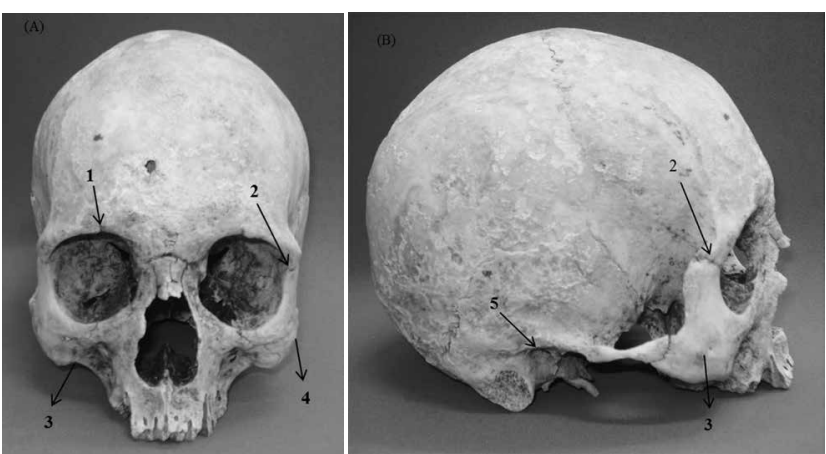

Figura 1 - (A) Foto frontal com pontos craniométricos. (B) Foto perfil com pontos craniométricos - 1: Forame supra orbitário (FSO); 2: Sutura frontozigomática (Fz); 3: Pilar maxilo-zi gomático (PMZ); 4: Extremidade lateral do arco zigomátiCo $(Z y)$; 5: Meato acústico externo (MAE),

\section{Confecção de defeitos ósseos}

A partir do crânio seco foram realizados aleatoriamente dois defeitos ósseos (na região frontal direita e zigomática esquerda) com a finalidade de simular um acidente traumático no complexo maxilofacial com auxílio de micromotor cirúrgico da marca Beltec ${ }^{\circledR}$ (Araraquara, São Paulo, Brasil) e brocas de carboneto de tungstenio 701 e 702 Jet $^{\circledR}$ (Morrisburg, Ontário, Canadá).

\section{Exames de imagem e prototipagem}

Foram realizados exames de imagem tipo tomografia computadorizada (Fig. 2- A e B) no Serviço de Radiologia do Hospital da Cidade de Passo Fundo - RS, com a finalidade de mensurar o defeito ósseo e também para confeccionar o protótipo que foi realizado para projetar a prótese individualizada.

As imagens de tomografia computadorizada foram obtidas com espaçamento máximo de $1 \mathrm{~mm}$ entre as fatias e salvas em formato Dicom. Ao todo, foram realizadas quatro tomografias: uma anterior à confecção do defeito ósseo; outra após a confecção do defeito, a terceira dos protótipos e das porções removidas do crânio seco e a última após a instalação da prótese.
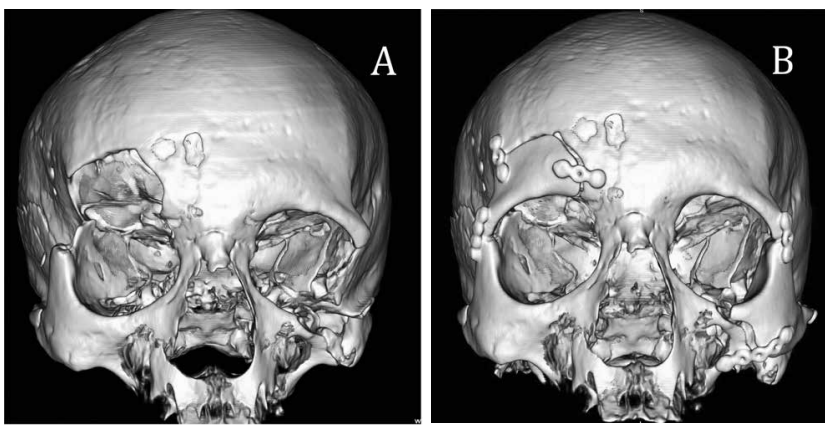

Figura 2 - Imagem de reconstrução volumétrica realizado a partir da tomografia computadorizada: (A) após confecção dos defeitos ósseos (B) após instalação das próteses

A imagem de tomografia computadorizada foi transformada em um modelo tridimensional utilizando o software InVesalius ${ }^{\circledR}$ (Centro de Pesquisas Renato Archer - CenPRA - Ministério de Ciência 
e Tecnologia, Brasil). O modelo tridimensional resultante foi salvo em formato STL, arquivo padrão reconhecido por equipamentos de prototipagem rápida. Tal padrão de arquivo representa uma superfície tridimensional composta por uma malha de triângulos. O arquivo STL, representando o crânio do paciente, foi importado no software Geomagic Studio $12^{\circledR}$ (Raindrop Geomagic, Inc., EUA). O software permite a visualização e a edição de arquivos de superfície. Utilizando uma ferramenta do software, foi realizada uma operação de espelhamento em relação ao eixo vertical (plano Y-Z) do crânio do paciente. Após ser realizado o espelhamento os dois arquivos (normal e espelhado) foram sobrepostos. Como resultado de uma intersecção entre os dois modelos restam as partes faltantes do crânio original do paciente (órbita e zigoma) (Fig. 3).

A confecção do protótipo das próteses de reconstrução foi feita pela técnica de prototipagem rápida, técnica na qual a imagem virtual do crânio seco (obtida através da tomografia computadorizada) é transformada em um modelo físico, que representa a réplica do crânio seco sob investigação.

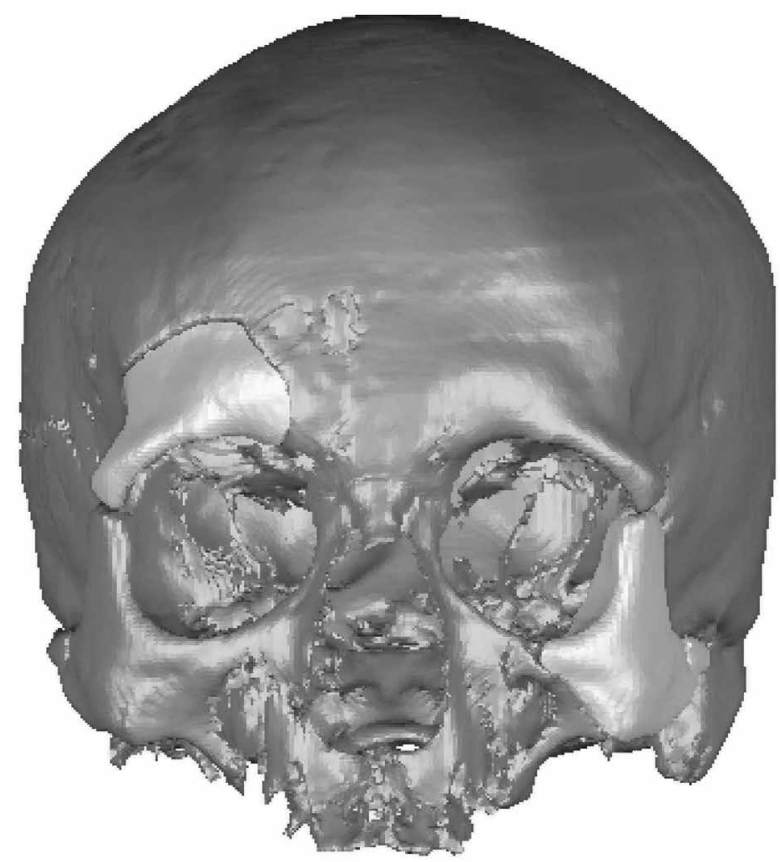

Figura 3 - Próteses instaladas e confeccionadas por método de espeIhamento para o osso frontal e zigomático

\section{Próteses}

Os protótipos das próteses foram fabricados no Laboratório de Inovação Tecnológica da Faculdade de Engenharia Mecânica da Universidade de Passo Fundo pela técnica de impressão tridimensional (3DPTM).

O equipamento utilizado é do tipo 3DPTM, modelo Z310 Plus, de fabricação espanhola pela empresa Z Corporation ${ }^{\circledR}$ (Rock Hill, South Carolina, EUA) e tem seu princípio de funcionamento semelhante a uma impressora jato de tinta, que utiliza cartuchos iguais aos usados em impressoras da marca Hew-
lett-Packard ${ }^{\circledR}$ (HP, Palo Alto, Califórnia, EUA), sendo que os mesmos foram esvaziados e alimentados com resina. $\mathrm{O}$ formato de arquivo utilizado e aceito para impressão foi STL.

A impressora possui dois recipientes, nos quais, de um lado fica o pó a ser aplicado e do outro, o local onde será impresso o modelo. O processo se dá em camadas, sendo que há uma espécie de cabeçote no qual estão inseridos os cartuchos alimentados por uma resina aglutinante. Essa resina fica armazenada em um recipiente na parte superior. $\mathrm{O}$ cabeçote tem um movimento livre (eixos transversais e longitudinais) podendo, então, executar o desenho a cada camada da peça com spray de resina e, logo após, o equipamento recolhe uma camada de pó (aproximadamente $0,0875 \mathrm{~mm}$ ) do outro lado, e o deposita sobre a resina, e assim sucessivamente, ou seja, o modelo vai sendo impresso em pequenas camadas de resina e pó, de baixo para cima. Portanto, teoricamente, quanto maior o modelo, mais camadas necessárias.

Para executar o processo foi utilizado como material Resina precisão Z- Bond TM 101; Cola - Zb 60 e Pó Zp 131 (Z Corporation ${ }^{\circledR}$, Rock Hill, South Carolina, EUA). Com o software, foi usado uma extensão STL para originar os comandos para a execução da peça. Para a impressão obter uma melhor qualidade e um resultado mais preciso, a temperatura de trabalho esteve em torno dos $38^{\circ} \mathrm{C}$ e se teve uma umidade do ar nos limites de $20 \%$ a $40 \%$. Depois de finalizado o processo de impressão a peça ainda permaneceu imersa no pó por 60 minutos e somente após esse período foi possível removê-la para que fosse efetuada a limpeza e a aplicação da cola Z-Bond, a qual auxilia no endurecimento da peça.

\section{Avaliação após confecção do protótipo da prótese}

Apos a confecção do protótipo das próteses, essas foram fixadas no crânio seco com a utilização de microplacas e microparafusos (Fig. 4) e para avaliação da fidelidade do processo de reprodução foram utilizados os seguintes recursos: mensuração, fotografias, tomografias e análise gráfica.
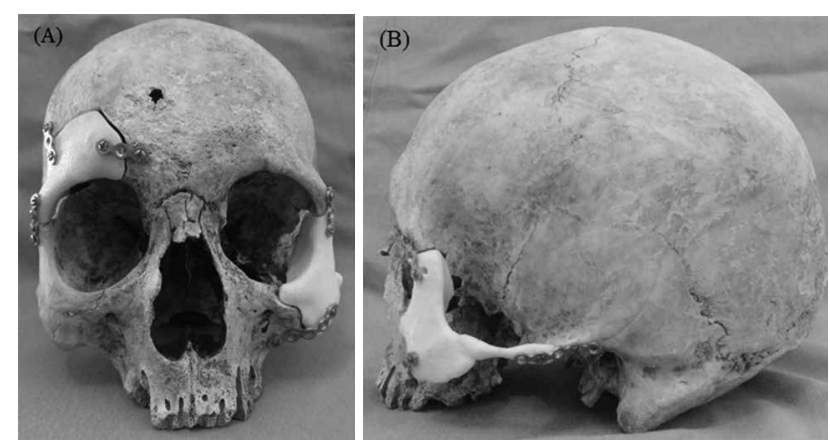

Figura 4 - Aspecto final do crânio seco com as próteses fixadas. (A) Vista frontal e (B) vista lateral 


\section{Resultados}

\section{Medidas}

Após a realização das medidas manualmente (Fig. 5), - foram obtidos os resultados que podem ser visualizados na Tabela 1 , a qual identifica os valores adquiridos na mensuração do crânio seco anterior à confecção dos defeitos ósseos, sendo que o valor de cada coluna foi obtido a partir de uma média das dez repetições que foram realizadas em cada segmento. A mensuração - Pós reconstrução - foi feita após a instalação das próteses. Para fins comparativos, foram excluídos dois itens desta tabela, PFSe e ZMAd, pois não haveria mudanças dimensionais uma vez que as próteses foram colocadas nos lados opostos a estes locais e tais resultados não alterariam.

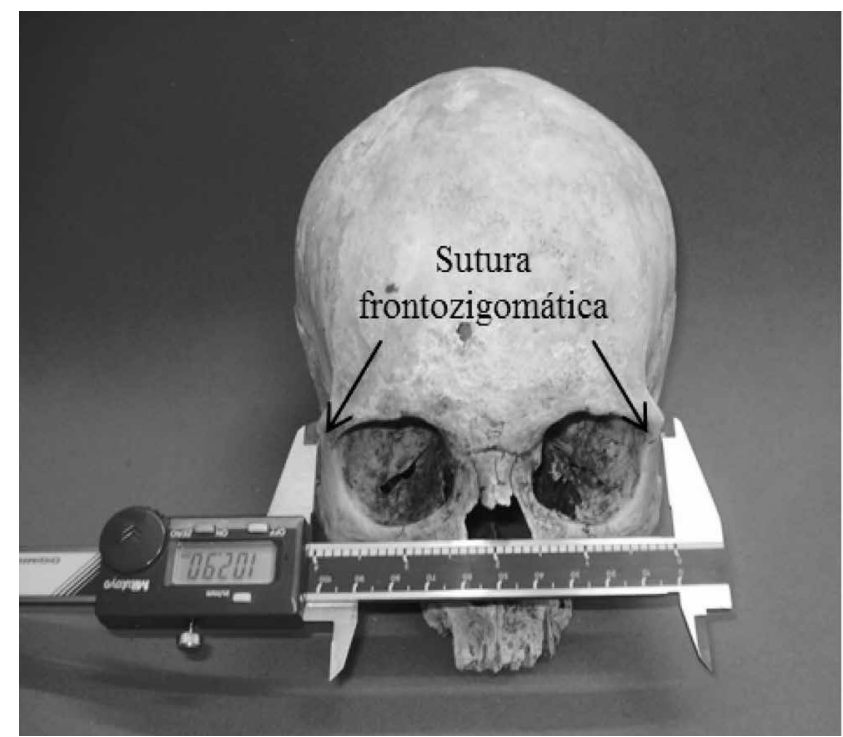

Figura 5 - Imagem ilustrativa da forma de mensuração do crânio com paquímetro digital

Comparando os valores desta tabela nota-se que não houve diferença significativa nas médias finais, sendo que a maior diferença mostra-se na medida PFSd de 0,97 $\mathrm{mm}$, enquanto que a menor aparece na medida LBZ de 0,06 mm (Tabela 1 ).

Tabela 1 - Comparação das médias das medidas ( $\mathrm{mm}$ ) do crânio seco antes e após a confecção dos defeitos e adaptação das próteses, sendo considerado valor positivo para o aumento de volume e negativo para a redução

\begin{tabular}{l|c|c|c|c}
\hline \multicolumn{1}{c|}{ Medidas } & LBZ & FZ-FZ & PFSd & ZMAe \\
\hline Pré defeito & 107,83 & 102,20 & 60,17 & 75,67 \\
Pós reconstrução & 107,89 & 102,57 & 59,20 & 75,29 \\
Diferença & $\begin{array}{c}+0,06 \\
(0,05 \%)\end{array}$ & $\begin{array}{c}+0,37 \\
(0,36 \%)\end{array}$ & $\begin{array}{c}-0,97 \\
(1,6 \%)\end{array}$ & $\begin{array}{c}-0,38 \\
(0,5 \%)\end{array}$ \\
\hline
\end{tabular}

\section{Exames de imagem e prototipagem}

Com a utilização do software Geomagic Qualify $12^{\circledR}$ (Raindrop Geomagic, Inc., EUA) foi comparado graficamente por sobreposição de imagem o volume físico da prótese e da peça originalmente subtraída do crânio. Foram obtidos gráficos em cores, representando o desvio tridimensional para cada biomodelo. Nesses gráficos estão na cor verde as regiões cujo desvio é tão pequeno que é desprezível $(+0,4$ a $-0,4 \mathrm{~mm})$; em tons de vermelho e amarelo são as regiões em que ocorreu aumento de volume, ou seja, com desvio positivo (+0,5 a $2,2 \mathrm{~mm}$ ) e em tons de azul e roxo são as regiões nas quais ocorreu redução de volume, o que corresponde a um desvio negativo (-0,5 a 2,2mm).

Observa-se que as peças do osso frontal sobrepostas apresentam uma região avermelhada nas extremidades seguindo para o centro em tom alaranjado (Fig. 6A). Contudo, na Figura 6B por uma vista interna, prevalecem os tons de verde em que o desvio é praticamente inexistente. Em ambas as figuras, o desvio padrão foi de aproximadamente $0,76 \mathrm{~mm}$.

Na Figura 6C (zigomático), notam-se áreas esverdeadas e azuladas, ou seja, houve uma variação de aproximadamente $-0,5$ a $+0,5$, sendo que a média de desvio padrão ficou em torno de $0,6 \mathrm{~mm}$.

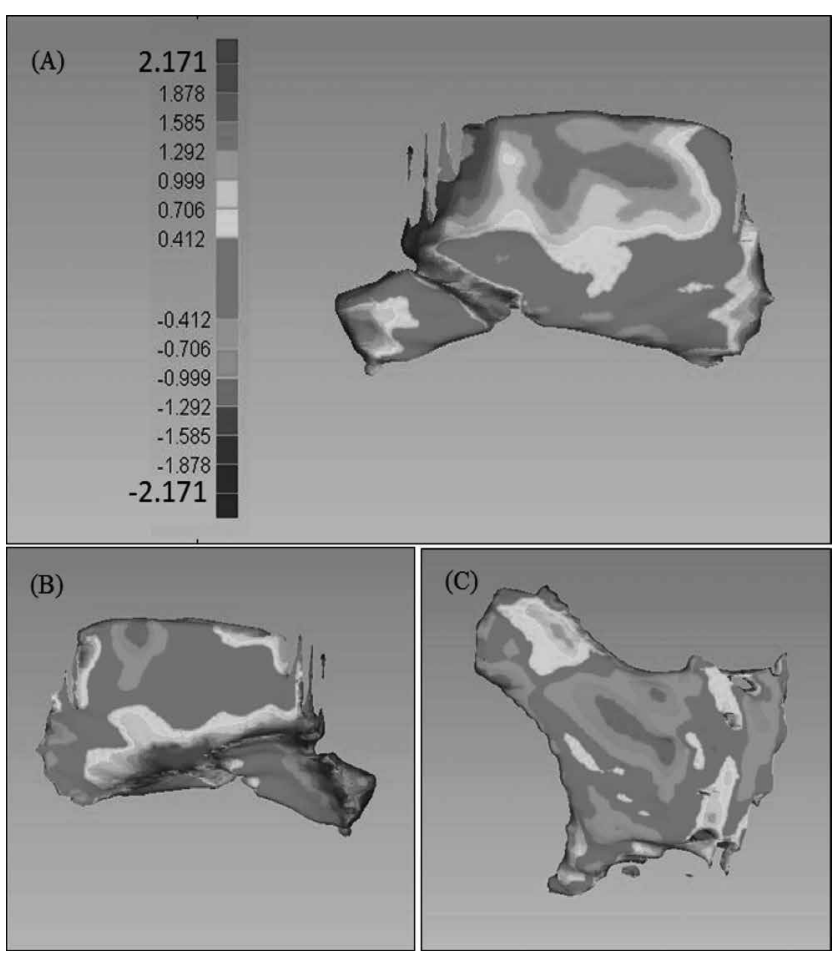

Figura 6 - Sobreposição das imagens tomográficas pelo software Ge omagic Qualify 12® para avaliar o desvio tridimensional de cada prótese. A) Osso Frontal face externa; B) Osso Frontal face craniana; C) Osso Zigomático face medial 


\section{Discussão}

Os processos de aquisição de imagens biomédicas registraram avanços exponenciais nas últimas décadas, como pode ser exemplificado com as tomografias computadorizadas. Equipamentos cada vez mais sofisticados de TC ou RNM geram imagens tridimensionais de alta qualidade e fornecem informações detalhadas sobre o paciente 1,4,12,13$^{\text {. }}$.

A Prototipagem Rápida é um processo de tecnologia multidisciplinar que consiste na produção de modelos em três dimensões, obtidos a partir de imagens de Tomografia Computadorizada (TC) e Ressonância Nuclear Magnética (RNM). É uma ferramenta auxiliar para tratamento de defeitos e neoplasias craniofaciais e que fornecem dados suficientes para a produção de biomodelos de estruturas ósseas, os quais, na maioria dos casos, são suficientes para a realização dos planejamentos e procedimentos a serem realizados ${ }^{2,4}$.

Devido ao seu caráter físico, biomodelos são mais fáceis de interpretar e manipular do que imagens de TC e RNM ${ }^{4,12-14}$. Isso faz deles uma ferramenta útil nos casos cirúrgicos mais complexos, permitindo um melhor planejamento e uma simulação prévia, além de facilitar a comunicação com o paciente ${ }^{14,15}$. Além disso, uma aplicação dos biomodelos que vêm ganhando destaque é o design e a fabricação de implantes e próteses personalizadas, em que um protótipo da prótese é moldado sobre o modelo para sua posterior fabricação. Estudos apontam o potencial dos biomodelos em reduzir o custo global do tratamento, além de conduzir a melhores resultados ${ }^{7}$.

A prototipagem rápida tem se constituído em um recurso de grande importância para o planejamento cirúrgico-protético de situações de alta complexidade, como as vivenciadas na implantodontia contemporânea. $\mathrm{O}$ estudo diagnóstico com o uso de biomodelos pode ser recomendado, pois reduz o custo global do tratamento, elimina potenciais erros clínicos e conduz a melhores resultados. Dessa forma, é possível utilizar a prototipagem para obter próteses de titânio, possibilitando que peças complexas possam ser construídas com grande precisão, para serem substitutas das regiões ósseas perdidas ${ }^{16,17}$.

Os processos de prototipagem mais utilizados para confecção de protótipos com fins odontológicos são a estereolitografia, a sinterização a laser seletivo, a modelagem por fusão e deposição e a impressão tridimensional. Dentre esses métodos, o de menor custo é o 3D Print (Impressão Tridimensional) que é o processo de prototipagem com menor precisão dimensional e menor reprodução de estruturas finas ${ }^{2,7}$. A provável causa disto é a etapa de pós-processamento do modelo, na qual ocorre a adição de resina ao modelo feito em gesso para lhe conferir mais resistência mecânica, causando sua expansão.
Cada processo confecciona um modelo com características particulares, que devem ser analisadas no momento de se realizar o pedido de um biomodelo. O fim para o qual o protótipo esta sendo solicitado deve ser levado em conta para que se possa tirar o melhor proveito possível dessa tecnologia. Mas, por mais que se escolha a opção mais adequada, ainda existe a barreira da pouca disponibilidade de equipamentos voltados para área da saúde.

O 3DPTM, embora também não use material de suporte, necessita de uma etapa de pós-processamento inexistente nos demais, que envolve a infiltração de resina no modelo para lhe conferir maior resistência mecânica. O processo FDM, por exemplo, exige como etapa de pós-processamento, a remoção dos suportes. Quanto aos tempos, destaca-se negativamente o grande tempo de fabricação observado para o processo FDM, o qual totaliza cerca de um dia e meio. Já o processo 3DPTM destaca-se por ser mais rápido, com a vantagem adicional de ser o de menor custo dentre os processos de prototipagem rápida. Nos resultados desta análise, o 3DPTM é o único comparável aos processos de usinagem por comando numérico computacional $(\mathrm{CNC})^{18}$.

$\mathrm{O}$ processo da engenharia de digitalização $3 \mathrm{D}$ pode ser usado para capturar a morfologia de partes deficientes ou normais do corpo humano, bem como imprimir um modelo físico. Nesse processo alguns pontos cegos podem ocorrer durante a aquisição da imagem, os quais necessitam ser preenchidos manualmente, porém esse sistema, que combina aquisição de imagem por TC com um software de modelamento biomédico, pode corrigir os detalhes anatômicos e ser aplicado com sucesso. Esse método se constitui como uma solução alternativa em relação ao custo - efetividade para reconstrução de defeitos ósseos ${ }^{2,19}$.

Os desvios encontrados em todo o processo deste estudo, desde a aquisição das imagens até a confecção por usinagem, podem, em parte, ser creditados às técnicas de medição empregadas. Dessa forma, este trabalho comprova que a técnica da prototipagem é possível de ser aplicada na área médica. Entretanto, uma análise mais completa faz-se necessária para separar a variância inerente à medição daquelas induzidas pelos processos de aquisição, vetorização, reconstrução e usinagem. As hipóteses de acrescentar mais de um crânio à amostra e também o número de observadores podem comprovar a reprodutibilidade e a confiabilidade desta proposta de estudo piloto. Não obstante, os desvios totais são inferiores aos valores reportados por outros autores quando da aplicação da fabricação de próteses por prototipagem rápida ${ }^{16,18}$. 


\section{Conclusão}

Este estudo piloto revela que o método de reconstrução de defeitos ósseos com biomodelo obtido por espelhamento gráfico e confecção por prototipagem rápida pode ser uma alternativa terapêutica viável por apresentar fiéis reconstruções, uma vez que este método utiliza o próprio indivíduo como modelo. A busca por um material biocompatível que possa ser colocado diretamente na prototipadora deve ser testada para assim constituir uma técnica ideal para reconstrução de partes do corpo humano perdidas de forma traumática.

\section{Abstract}

Rapid Prototyping has proved to be a very useful tool to aid the development of new products in the biomedical field. This technology allows obtaining physical prototypes from the conversion of $X$-ray computed tomography images. Objective: The aim of this pilot study was to test the accuracy of the prototyping process by three-dimensional printing technology. Materials and method: Biomodels were made from customized prosthesis produced by the reverse engineering design method for the reconstruction of bone defects in the craniofacial complex, created on human dry skull. Results: Comparing both measures, there was neither significant difference nor a considerable deviation when the original bones were graphically compared to their respective prototypes. Conclusion: This study shows that the reverse engineering design method for obtaining a biomodel, and its creation by using Rapid Prototyping may be a viable therapeutic alternative, considering this method offers faithful reconstructions for using patients themselves as models.

Keywords: Tomography. Three-dimensional imaging. Anatomic models.

\section{Referências}

1. Tepper OM, Sorice S, Hershman GN, Saadeh P, Levine JP, Hirsch D. Use of virtual 3-dimensional surgery in post-traumatic craniomaxillofacial reconstruction. J Oral Maxillofac Surg 2011; 69:733-41.

2. Silva DN, Oliveira MG, Meurer E, Meurer MI, Silva JVL, Santa-Bárbara A. Dimensional error in selective laser sintering and 3D-printing of models for craniomaxillary anatomy reconstruction. J Cranio-Maxillofac Surg 2008; 36(8):443-9.

3. Milovanovic J, Trajanovic M. Medical applications of rapid prototyping. Facta Universitatis: mechanical engineering 2007; 5(1):79-85.

4. James WJ, Slabbekoom MA, Edgin WA. Correction of congenital malar hypoplasia using stereolithography for presurgical planning. J Oral Maxillofac Surgery 1998; 56(4):512-7.

5. Sailer HF, Haers PE, Zollicofer CPE. The value of stereolithographic models for preoperative diagnosis of craniofacial deformities and planning of surgical corrections. Int $J$ Oral Maxillofac Surg 1998; 27(5):327-33.
6. Peckitt NS. Stereoscopic lithography: customized titanium implants in orofacial reconstruction. Br J Oral Maxillofac Surg 1999; 37(5):353-69.

7. Meurer MI, Meurer E, Silva JVL, Barbara AS, Nobre LS, Gerhardt de Oliveira M, et al. Aquisição e manipulação de imagens por tomografia computadorizada da região maxilofacial visando à obtenção de protótipos biomédicos. Radiol Bras 2008; 41(1):49-54.

8. Steck JH, Miquelino R, Souza ALG, Gouveia MF, Silva JVL. Planejamento em cirurgias complexas em cabeça e pescoço - o uso de modelos tridimensionais. Rev Bras Cir Cabeça e Pescoço 2007; 36 (3):171-3.

9. Gorni AA. Introdução a prototipagem rápida e seus processos. Rev Plást Ind 2001; 31(3):230-9.

10. Selhorst Junior A. Análise comparativa entre os processos de Prototipagem Rápida na concepção de novos produtos [Dissertação de Mestrado]. Curitiba: Universidade Católica do Paraná; 2008.

11. Winder J, Bibb R. Medical Rapid Prototyping Technologies: State of the Art and Current Limitations for Application in Oral and Maxillofacial Surgery. J Oral Maxillofac Surg 2005; 63:1006-15.

12. Costa Filho CFF, Moura LA, Costa MGF. Revisão sobre métodos de segmentação 2D do ventrículo esquerdo. Rev Bras Eng Biom 1999; 15(3):141-58.

13. Brogdon BG. Definitions in forensics and radiology. Crit Rev Diagn Imaging 2000; 41(1):1-12.

14. Fogaça ACM, Menezes JDS, Gerhardt de Oliveira M, Hellwig I, Zerbinati LPS, De Conto F, et al. Análise de Biomodelos após autoclavagem. Ver Odontologia (ATO) 2011; 3:412-20.

15. Shoi SH, Samavedan S. Modelling and optimization of rapid prototyping. Comput Ind 2002; 47(1):39-53.

16. Choi JY, Choi JH, Kim NK, Kim Y, Lee JK, Kim MK et al., Analisys of errors in medical rapid prototyping models. Int $J$ Oral Maxillofac Surg 2002; 31:23-32.

17. Singare S, Lian Q, Wang WP, Wang F, Liu Y, Li D, et al. Rapid prototyping assisted surgery planning and custom implant design. Rapid Prototyping J 2009; 15(1):19-23.

18. Rockenbach M, Bertol LS, Silva FP, Roldo L. Avaliação da precisão e da facilidade de fabricação de diferentes métodos de produção de protótipos. In: V Congresso Internacional de Pesquisa em Design (CIPED): 2009; Bauru. Anais. V Congresso Internacional de Pesquisa em Design; 2009. p.1075-83.

19. Ferraz EG, Andrade LCS, Santos AR, Torregrossa VR, Freire MRS, Sarmento VA. Effect of different surface processing protocols in three-dimensional images for rapid prototyping. Adv Eng Softw 2011; 42:332-5.

Endereço para correspondência:

Ferdinando De Conto

Faculdade de Odontologia, - Universidade de Passo Fundo - RS

Campus I - Km 171 - BR 285 - Bairro São losé

Caixa Postal 611

99001-9470 Passo Fundo/RS

Fone: 5433168403

E-mail: ferdi@upf.br

Recebido: 11/11/2013. Aceito: 02/05/2014. 